\title{
Planificación y estrategia de identidad corporativa en los clubes deportivos españoles
}

Guillermo Sanahuja Peris | gsanahuj@uji.es

UNIVERSIDAD JAUME I

Resumen: La presente investigación trata de presentar un diagnóstico original sobre los procesos de planificación estratégica de la identidad corporativa en los clubes deportivos profesionales españoles. La metodología empleada es una combinación de técnicas cuantitativas y cualitativas ante un universo de estudio formado por las cincuentas seis entidades deportivas que forman la Liga de Fútbol Profesional y la Asociación de Clubes de Baloncesto.

Palabras clave: Comunicación corporativa, identidad corporativa, fútbol, baloncesto

Abstract: The aim of this paper is to show an original diagnosis about the planning process and corporate identity strategy in professional Spanish sport clubs. The methodology used is a combination of quantitative and qualitative techniques applied to a corpus of 56 sport clubs integrated in the Spanish Football Professional League (Liga de Fútbol Profesional) and Spanish Basketball Association Club (Asociación de Clubes de Baloncesto).

Key words: Corporate communication, corporate identity, football and basketball 


\section{Introducción: el interés de esta investigación}

La academia ha mantenido históricamente una distancia respecto a lo que acontecía en los terrenos de juego y en sus despachos colindantes. Los albores del siglo XXI han deparado el desarrollo inusitado y exponencial de las entidades deportivas en el territorio español. De forma paralela, en los últimos años existe una aproximación al conocimiento científico en el cruce de comunicación, deporte y marketing. Dicha aproximación se manifiesta básicamente a través de cinco vías: los grados en educación física, los másteres universitarios en gestión de entidades deportivas y foros en la Red $y$, finalmente, los centros de investigación específicos y la labor divulgativa de ciertos autores.

Este desarrollo y crecimiento de la industria del deporte profesional español no ha llegado a su fin. La gestión de las entidades deportivas aún presenta una distancia considerable, en conocimiento y empleo de herramientas, respecto a sus equivalentes en la empresa privada. Por tanto, la investigación presenta la motivación de aportar conocimiento a un sector en continuo crecimiento.

\section{El marco teórico}

\subsection{Historia y evolución de la comunicación y el marketing en los clubes deportivos}

A partir de una intensa labor documental y hemerográfica la presente investigación ha conseguido construir un relato histórico sobre la evolución de la comunicación y el marketing en los clubes deportivos. Desde los años 60 se dan algunas anecdóticas acciones que revelan la potencialidad de esta dimensión del deporte. En los años 70 se manifiestan las pioneras iniciativas como las primeras publicidades en camisetas del Joventut de Badalona o los primeros gabinetes de prensa al servicio de clubes deportivos. Los años 80, a la sombra del primer gran acontecimiento deportivo en España como fue el Mundial 82, suponen el inicio de la transformación global de unos clubes totalmente amateurs hacia la profesionalización. Nacen la ACB y la LFP. La voraz necesidad de ingresos fomentó el florecimiento de las estructuras comerciales adosadas a los clubes generalizándose la explotación comercial de los principales activos de una entidad como el naming right, la publicidad en la camiseta o las vallas perimetrales.

La transformación de clubes elementales en sociedades anónimas deportivas en los años 90 revoluciona el sector. La Ley del Deporte o la celebración de los JJOO de Barcelona 1992 o el crecimiento de la economía española contribuyeron al nacimiento y expansión de los departamentos de comunicación y marketing en los clubes deportivos. Las webs corporativas, el inicio de concesiones de licencias de producción y explotación de merchandising, los derechos de retransmisión o las zonas VIP corresponden a esta década. Se puede decir que la dirección de comunicación y marketing tal y como se entiende en la actualidad nace en esta etapa. 
El siglo XXI da la bienvenida al marketing deportivo. Mientras que los grandes clubes sofistican sus actuaciones en esta materia los clubes modestos se afanan en consolidar sus áreas funcionales de comunicación y marketing asignando recursos humanos de forma permanente. Sin embargo, el crecimiento de las organizaciones deportivas no evita el constante déficit financiero. La constante huida hacia delante constata un comportamiento irracional e infantil. Así lo mantiene la tribuna académica: Campos (2006), Barajas (2007) o Gómez y Opazo (2007), entre otros, indican las principales disfunciones en la gestión de las organizaciones deportivas: desorganización y carácter amateur de los ejecutivos; dirección paternalista, basada en las estructuras familiares y ausencia de investigación; toma de decisiones irracionales; la singularidad del mercado, el producto y la complejidad de sus actores; la presión mediática; el divismo de los presidentes; y un modelo de negocio insostenible con disparidad de dimensiones y basado en una distribución no equitativa de los ingresos por derechos de retransmisión y dependencia sobre éstos.

En los últimos años se ha estrechado el cerco sobre la nueva disciplina directiva de comunicación y marketing en las organizaciones deportivas encontrándose los trabajos de Arceo Vacas (2003), Olabe (2009) y Ginesta (2009). Olabe destaca en su investigación "la progresiva profesionalización del área de comunicación" y que el propósito fundamental de los departamentos de comunicación de los clubes es "mantener relaciones comunicativas con los mass media". El tercero, por su parte, certifica la heterogeneidad en la implantación de las TIC en el fútbol profesional o la indefinición de las competencias del director de comunicación.

\subsection{El corporate y la comunicación en las organizaciones}

La referencia académica o raigambre teórica de esta investigación se ubica precisamente sobre ese paradigma del siglo XXI y, en definitiva, sobre el corporate, definido como la moderna concepción de la gestión estratégica de la comunicación corporativa. El modelo de organización que plantea esta filosofía de management confía profundamente en la gestión de los recursos y activos intangibles como vectores diferenciales entre corporaciones (López Triana Y Sotillo, 2009), en la definición y planificación de una identidad corporativa (CAPRIOTTI, 2009), asumiendo la tres esferas de la comunicación (institucional, mercadológica e interna) que vindica Costa (2008), como paso imprescindible hacia el alcance de una imagen positiva, en la configuración de una reputación (Villafañe, 2004), en la figura del "DirCom" o el "CRO" (Chief Reputation Officer), ubicado en la cúspide de la organización, como integrador de todas las emisiones de mensajes, velador del capital relacional y como representante de los públicos ante la organización (Mut Camacho, 2010), en una integración de la comunicación en la organización (López Lita, 2000) y en una apuesta del largo plazo a partir de una planificación estratégica y de un desarrollo de las sociedades sostenible y respetuoso con sus grupos de interés. Los conceptos centrales de esta filosofía de management son la identidad, la imagen, la cultura corporativa, la reputación, la responsabilidad social corporativa y la marca, entendida ésta como la promesa de una experiencia que provee la organización (Alloza y Oliver, 2009). 


\subsection{La planificación estratégica y la integración de comunicación y marketing en las entidades deportivas}

Cualquier proceso directivo en una organización tendrá mayor probabilidad de culminarse con éxito si es el resultado de un plan estratégico (Pérez González, 2001). La distinción entre plazos, estrategia, táctica y acción es imprescindible en la gestión de empresas y entidades en la actualidad. Por tanto, el establecimiento de objetivos, la elección de una estrategia, su ejecución y la evaluación son cuatro pasos ineludibles en cualquier ámbito organizacional.

El proceso estratégico y la definición de una visión corporativa obligan a coordinar las distintas áreas de trabajo. Morales Serrano (2006: 340) señala que "la comunicación planificada, engloba a todas las teorías que estudian aquellas ocasiones en las que la comunicación es usada de modo consciente para buscar una serie de objetivos más o menos específicos". Dicha planificación se puede plasmar en el Plan Estratégico de Reputación, Imagen y Comunicación. En el caso de los clubes deportivos la integración de las funciones de marketing y comunicación en una sola dirección es más necesaria si cabe. Maria Hopwood, induciendo desde el caso del cricket inglés en 2005, señala:

\footnotetext{
Cuando las relaciones públicas están sometidas al marketing las organizaciones son privadas de una de las dos funciones críticas. El departamento de relaciones públicas no debe estar sometido al departamento de marketing, debe ser independiente. Si no es posible, cuanto menos, las dos funciones deben ser conceptual y operacionalmente distintas entre ellas (2005:28).
}

Mientras que en otras organizaciones ambas funciones pueden estar separadas 0 subordinadas una sobre la otra, a pesar de que autores desde distintas tribunas recomiendan su absoluta coordinación (Slotd, Dittmore y Branvold, 2006; Wilcox, Cameron y Xifra, 2007; y Rodriguez Ardura, 2007), en esta investigación se considera oportuno integrar completamente en una sola dirección a las dos funciones. En este sentido, los distintos autores sugieren que la explotación comercial debe coordinarse junto a la Dirección de Comunicación puesto que la comercialización y generación de ingresos de una entidad deportiva donde la base del negocio es el espectáculo deportivo (Chadwick y Beech, 2004) y la propia identidad del club (Toyos y Agudo, 2003) asociada a contenidos informativos. Así, la gestión de la identidad y de la comunicación - bases del corporate- determinará la generación de recursos como dos caras de la misma moneda.

En esta gran área que incluye ambas funciones directivas existe un manual de técnicas de comunicación y marketing, una caja de herramientas y un repertorio de funciones específico. Es posible disponer una serie de técnicas de comunicación (relaciones informativas, medios propios, comunicación financiera, de crisis, de RSC, del líder, publicidad, patrocinio, eventos, comunicación interna), y marketing (captación de fondos, plataformas de fidelización, CRM y bases de datos), unas herramientas (Manual de Gestión de Imagen y Comunicación, un Mapa de Públicos, un Manual de Normas o Planes Anuales) y un número determinado de funciones a disposición de la dirección integrada de comunicación y marketing. El 
conocimiento y aplicación de cada una de las técnicas, herramientas y funciones, plasmado en áreas concretas de trabajo en el seno de la organización, constituye el corpus técnico de la filosofía del corporate. La correcta imbricación de estos tres sistemas contribuirá a la satisfacción de la visión de la corporativa y la consecución de recursos intangibles positivos.

\section{Objetivos, hipótesis, metodología y trabajo de campo}

\subsection{Objetivos}

Las principales finalidades de la presente investigación han sido las siguientes:

- Presentar un diagnóstico original sobre la planificación estratégica de la identidad corporativa en los clubes deportivos profesionales.

- Colaborar en la transformación del deporte profesional español con la argumentación de nuevos modelos de gestión orientados hacia la excelencia.

\subsection{Hipótesis}

De acuerdo con los precedentes expuestos, esta investigación se plantea la siguiente hipótesis: Los clubes deportivos profesionales no presentan una planificación estratégica de la identidad corporativa. Aunque disponen de valores, planes y estrategias no los explicitan y comparten con sus públicos. La construcción y respeto de la identidad propia contribuirá al entendimiento de la organización con sus distintos públicos y su reconocimiento institucional.

\subsection{Metodología}

Para llevar a cabo esta investigación se ha elegido el modelo de investigación propuesto por el catedrático Rafael López Lita (2001) en la publicación Las Agencias de Publicidad: evolución y posicionamiento futuro. El presente trabajo se articula en cuatro grandes etapas (la Etapa Exploratoria, la Etapa Cuantitativa, la Etapa de Contraste y Definitiva) que se explican en los siguientes epígrafes.

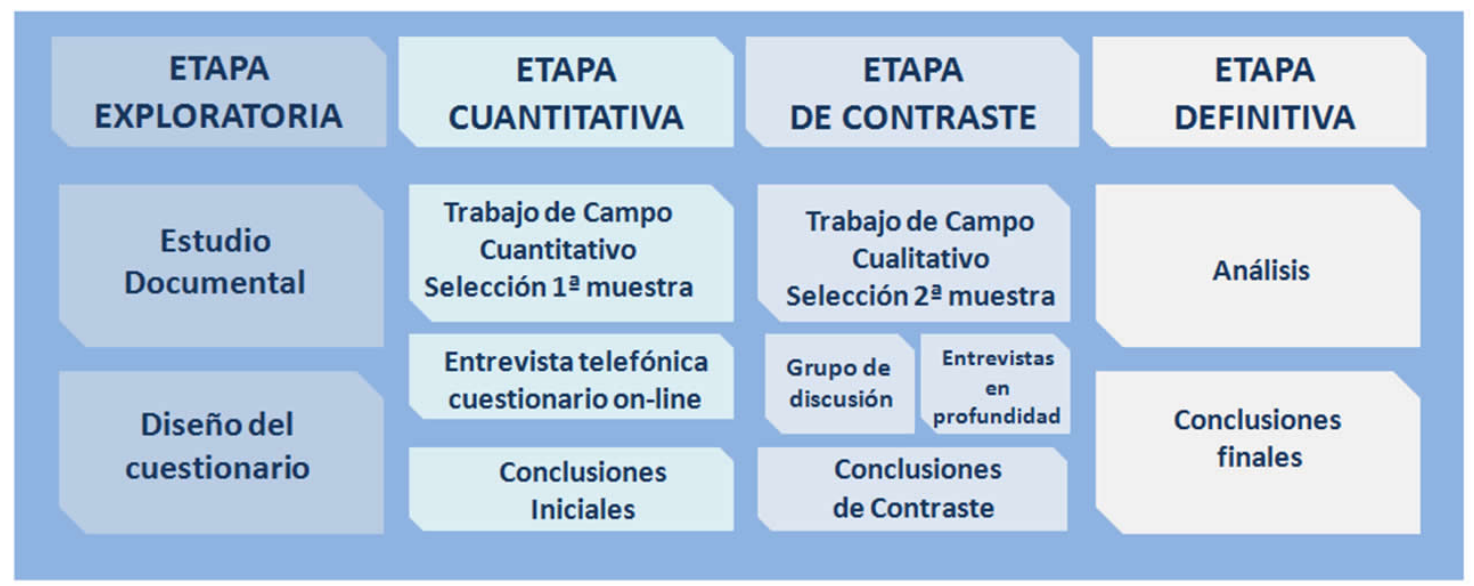


Figura I. Etapas de la investigación. Fuente: elaboración propia.

\subsubsection{Etapa Exploratoria}

La Etapa Exploratoria incluye a su vez dos fases:

A) Estudio Documental

Esta fase consiste en un estudio documental sobre las principales aportaciones teóricas en el ámbito de la comunicación corporativa, del marketing deportivo y de la gestión empresarial aplicada al deporte.

B) Diseño del cuestionario

A partir de investigaciones previas y fuentes documentales se reconoce la existencia de tres perfiles de competencias vinculados a las áreas de comunicación y/o marketing en los clubes deportivos profesionales: el Director de comunicación, el Director de marketing y el Director de comunicación y marketing. Esa diversidad de perfiles directivos en las áreas objeto de estudio invita al diseño de un cuestionario específico para cada uno de los tres perfiles.

\subsubsection{Etapa Cuantitativa}

A) Selección de la primera muestra

Partimos de un universo formado por 56 clubes que forman el tejido profesional de clubes de deportes de equipo en España, integrados en la LFP o ACB. De ellos, 38 son de fútbol, 16 de baloncesto y 2 clubes, el FC Barcelona y el Real Madrid, son polideportivos. La segunda división de fútbol o Liga Adelante cuenta con 22 clubes pero se contabilizan 20 clubes puesto que dicha competición participan los filiales del FC Barcelona y el Villarreal CF.

Puesto que esta etapa de la investigación es de corte cuantitativo y el universo de estudio no es inabarcable, se opta por igualar el universo a la muestra, es decir, se seleccionar como muestra a todo el universo de estudio: los 56 clubs que comprenden la LFP Y ACB.

B) Entrevista telefónica on-line

Esta primera fase del trabajo de campo duró aproximadamente dos meses. A partir de la buena disposición de los profesionales se ha alcanzado un índice de respuesta del 73,3 por ciento. Los cuestionarios han sido contestados por 77 de los 105 profesionales. Por colectivos o figuras profesionales, los directores de comunicación y marketing (7) han respondido en un 100 por ciento. 36 de 49 directores de comunicación ha respondido al cuestionario (73,4 por ciento) y en el caso de los directores de marketing han sido 34 sobre 49 (69,3 por ciento) los que han participado. 
Si se clasifica a los profesionales por la categoría en la que militan sus clubes, se recogen los siguientes índices de respuesta. La liga BBVA presenta una respuesta de 24 profesionales sobre 38 (63,1 por ciento). De los profesionales de la liga ADELANTE han respondido un total de 23 sobre 35 (65,7 por ciento). De la liga ENDESA de baloncesto, 30 de 32 profesionales $(93,75$ por ciento).

\begin{tabular}{|c|c|c|}
\hline $\begin{array}{c}\text { LIGA BBVA } \\
1^{\text {a }} \text { División de Fútbol }\end{array}$ & $\begin{array}{c}\text { LIGA ADELANTE } \\
\text { 2a División de Fútbol }\end{array}$ & $\begin{array}{c}\text { LIGA ENDESA } \\
\text { División de Honor Baloncesto }\end{array}$ \\
\hline Athletic Club de Bilbao & AD Alcorcón & Asefa Estudiantes \\
\hline Club Atlético de Madrid & CD Alcoyano & Assignia Manresa \\
\hline FC Barcelona & UD Almería & Baloncesto Fuenlabrada \\
\hline Real Betis Balompie & Cartagena CF & Bizkaia Bilbao Basket \\
\hline RCD Espanyol & Celta de Vigo & Blancos de Rueda Valladolid \\
\hline Getafe CF & Córdoba CF & CAI Zaragoza \\
\hline Granada CF & RC Deportivo de La Coruña & Caja Laboral Baskonia \\
\hline UD Levante & Elche CF & Cajasol Banca Cívica \\
\hline Real Madrid CF & Nàstic De Tarragona & FIATC Mutua Joventut \\
\hline Málaga CF & Girona FC & Gran Canaria 2014 \\
\hline RCD Mallorca & CD Guadalajara & Lagun Aro GBC \\
\hline CA Osasuna & Hércules CF & Lucentum Alicante \\
\hline Sevilla FC & SD Huesca & Obradoiro CAB \\
\hline Racing Santander & UD Las Palmas & UCAM Murcia \\
\hline Rayo Vallecano & Real Murcia CF & Unicaja Málaga \\
\hline Real Sociedad & CD Numancia & Valencia Basket \\
\hline Sporting de Gijón & CE Sabadell & FC Barcelona \\
\hline Valencia CF & Recreativo Huelva & Real Madrid CF \\
\hline Villarreal CF & Real Valladolid CF & \\
\hline \multirow[t]{3}{*}{ Real Zaragoza } & Xerez Deportivo & \\
\hline & FC Barcelona "B" & \\
\hline & Villarreal CF "B" & \\
\hline
\end{tabular}


Tabla I. Clubes que integran el universo de estudio.

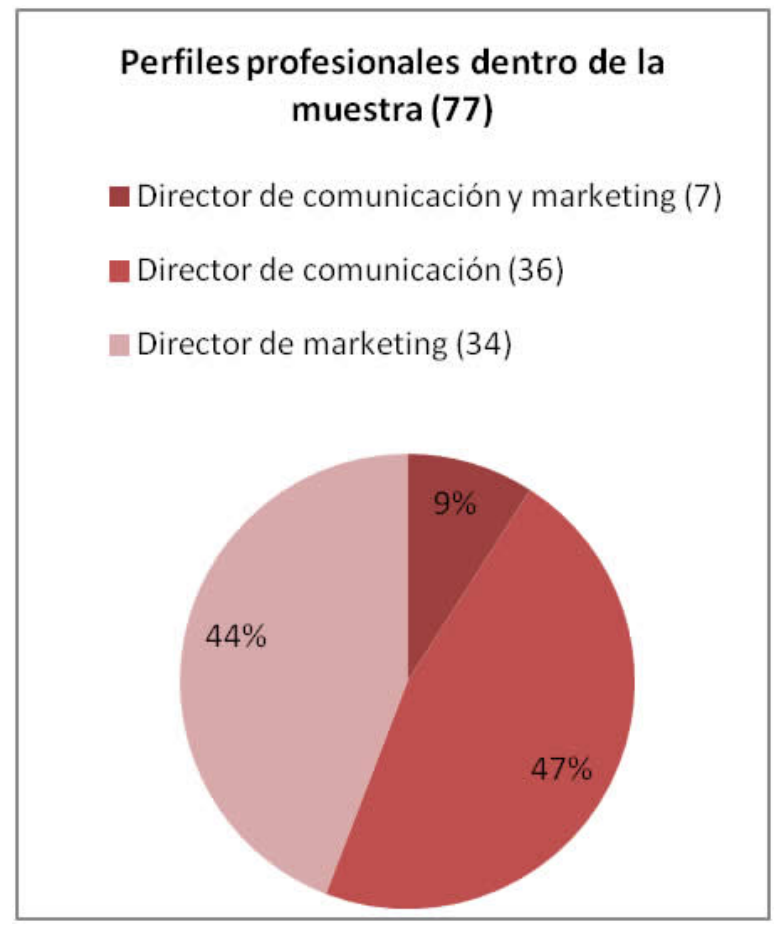

Figura II. Perfiles profesionales participantes. Fuente: elaboración propia.

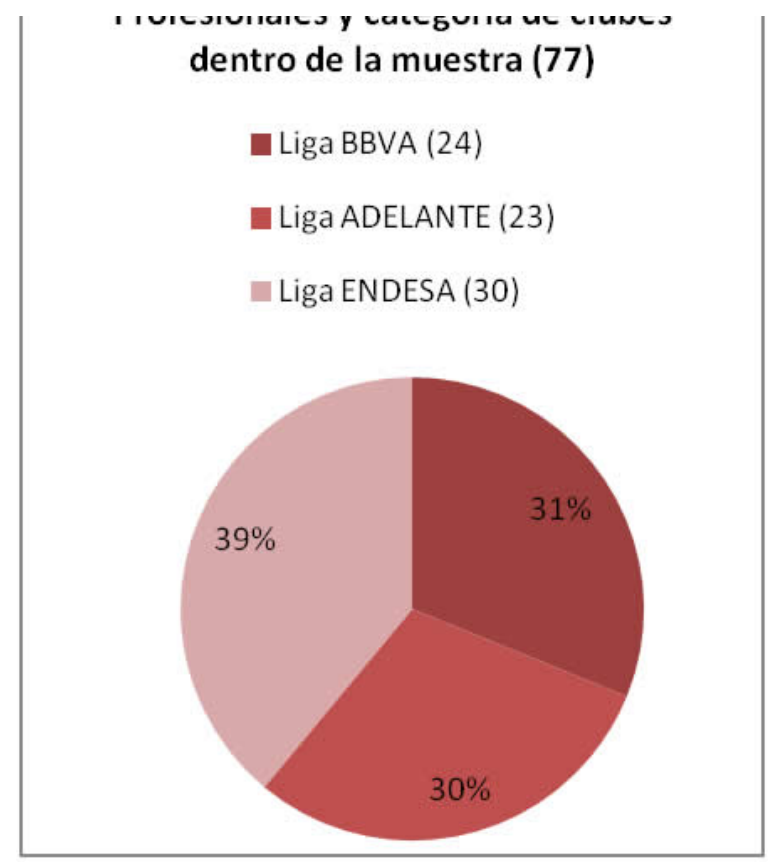

Figura III. Índice de respuesta de clubes deportivos. Fuente: elaboración propia. 
En cuanto a clubes, 39 de los 56 lo han hecho de forma completa, es decir, el director de comunicación y marketing o el director de comunicación y el director de marketing han respondido a sus respectivos cuestionarios. Seis clubes ha respondido de forma parcial: ha respondido el director de marketing o bien el director de comunicación. Y, finalmente, once clubes han declinado participar.

Así, han desestimado responder al cuestionario los siguientes clubes: Athletic de Bilbao, FC Barcelona, Betis Balompie, Málaga CF, Real Madrid, Real Sociedad, AD Alcorcón, CD Alcoyano, Cartagena CF, Hércules CF y Recreativo de Huelva. Cuatro de la primera división de fútbol, cinco de la segunda división y dos que, como se ha señalado, forman parte de la LFP y ACB. Excepto los dos clubes mixtos, el total de los clubes de la Liga ACB han tomado parte en la investigación.

C) Conclusiones iniciales

A partir de las entrevistas telefónicas on-line y la respuesta a los respectivos cuestionarios, se desarrolla un análisis general del estado de la comunicación y el marketing en los clubes deportivos profesionales españoles.

\subsubsection{Etapa de Contraste}

A) Selección de la segunda muestra.

Tras el establecimiento de unas conclusiones iniciales, de corte cuantitativo, sobre el estado de la cuestión y escenarios futuros, éstas se someten a un proceso de confrontación con el segundo trabajo de campo a partir de técnicas cualitativas (Álvarez-Gayou, 2003:128). Se eligen profesionales vinculados al mundo de la comunicación corporativa, la gestión empresarial, el marketing deportivo y el periodismo deportivo que a día de hoy no forman parte de ningún club profesional de fútbol y baloncesto. Se cuenta así con once profesionales de reconocido prestigio o "expertos". En esta etapa se opta por la combinación de dos técnicas de investigación cualitativas: el Grupo de discusión y una serie de Entrevistas en profundidad.

B) El grupo de discusión.

El espíritu dialéctico de un grupo de discusión (Báez y Pérez de Tudela, 2009:139) acoge la Con una duración aproximada de dos horas, durante la celebración del "focus group", se expone a través de una presentación en pantalla la información sujeta a debate por parte de los cuatro "expertos" integrantes del grupo. Posteriormente se registra y transcribe para el análisis de los resultados.

\begin{tabular}{|l|l|l|}
\hline Carlos Campos & Director & Managing Sport
\end{tabular}




\begin{tabular}{|l|l|l|}
\hline Sebastián Cebrián & Director General & DIRCOM \\
\hline Sandra Sotillo & Socia-Consultora & Villafañe y Asociados \\
\hline Pascual Martínez & Director estrategia y Desarrollo & Federación Española Baloncesto \\
\hline
\end{tabular}

\section{Tabla II. Integrantes del Grupo de Discusión.}

C) Las Entrevistas en profundidad.

Tras la revisión y contraste del estado, los retos y el modelo por parte de los "expertos" participantes en el "grupo de discusión", la segunda sub-fase de la etapa de contraste viene dada por la celebración de siete entrevistas en profundidad. Las entrevistas en profundidad (Báez y Pérez de Tudela, 2009:112) constan de un diseño específico a partir de un cuestionario semiestructurado basado y de una duración acotada entre los treinta y sesenta minutos.

\begin{tabular}{|l|l|l|}
\hline Antonio Lacasa & Consultor & AL Comunicación \\
\hline Amalio Moratalla & Director de Reputación y Deporte & Llorente \& Cuenca \\
\hline Ángel Alloza & CEO & Corporate Excellence \\
\hline Emilio Abejón & Coordinador & FASFE \\
\hline Manuel Saucedo & Director de comunicación & Unidad Editorial \\
\hline Ramón Fuentes & Redactor y presentador & Mediaset España \\
\hline Fco. Fernández Beltrán & Director & Dircom UJI \\
\hline
\end{tabular}

Tabla III. Entrevistados en Profundidad.

D) Conclusiones de contraste.

De las dos fases en las que consta el trabajo de campo cualitativo, se extrae a partir de un análisis de contraste en relación a los resultados, retos y modelo que se ha expuesto en la 
etapa documental y cuantitativa, unas conclusiones acumuladas y consecutivas de ambas sub-fases.

\subsubsection{La Etapa Definitiva}

La última se constituye sobre el análisis de los resultados globales de la investigación. Supone la extracción de los resultados de la misma, la verificación o refutación de hipótesis y la redacción de las conclusiones finales.

\section{Resultados de la investigación}

\subsection{Filosofía, planificación corporativa y planificación específica}

El primer bloque de los resultados hace referencia a las bases identitarias de los clubes deportivos. La primera de las preguntas incide en la misión y visión de los clubes deportivos. De forma global, una gran parte de los profesionales (77 por ciento) reconoce no disponer de forma explícita de una visión y misión. La fijación de unos valores propios u orientaciones estratégicas recoge unos datos muy similares (un 65 por ciento admite que no son explícitos).

Esa distribución se mantiene vigente ante la pregunta sobre el plan general estratégico. Un 70 por ciento reconoce que no es explícito. Resultado idéntico ante la pregunta sobre la existencia de una estrategia de comunicación y marketing vinculada: un 71 por ciento. En estas cuatro preguntas, la respuesta de mayor peso es "Sí, aunque no está redactada" (61 por ciento /61 por ciento /48 por ciento y 53 por ciento ).

No obstante, ante la pregunta "¿El departamento cuenta con unos objetivos específicos?" varia la tendencia de las anteriores preguntas, fundamentalmente en el área de marketing, puesto que sus objetivos pueden ser cuantitativos o numéricos. Lo cual modifica sensiblemente el gráfico global. En este caso, se iguala la explicitación con la presunción de esos objetivos (44 por ciento "sí, de forma explícita"/ 44 por ciento "sí, aunque no está redactada"). 
Índice de respuesta clubes (Universo: 56)

Clubes completos (39)

Clubes parciales (6)

Clubes ausentes (11)

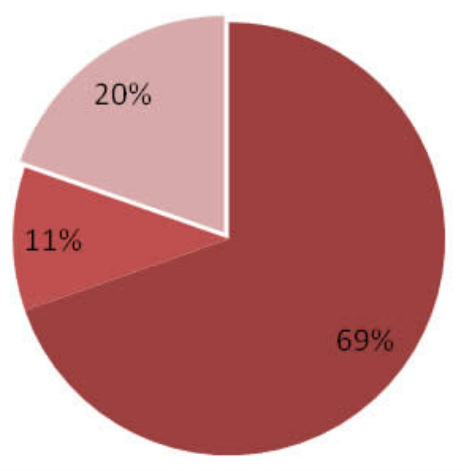

Figura 4. Filosofía, planificación y herramientas específicas estrategia de los clubes. Fuente: elaboración propia.

\subsection{Herramientas de planificación y estrategia}

El segundo de los bloques analiza a través de un cuadro similar al anterior el grado de empleo de las herramientas de gestión vinculadas a la estrategia de comunicación y/o marketing.

La plasmación de un Manual de Gestión de Imagen y Comunicación (MÁGICO) presenta una distribución equilibrada de las tres respuestas (34 por ciento, 34 por ciento y 32 por ciento) Así, un 66 por ciento acuerda no redactar esa planificación.

La Reputación Corporativa como concepto de trabajo, no ha penetrado todavía en el deporte. Tres de cada cuatro clubes no cuenta con un plan director de reputación. Un 17 por ciento señala que cuenta con él pero no está redactado. Un 6 por ciento de los profesionales, señalan disponer de ese plan.

La planificación de comunicación y/o marketing cuenta también con una respuesta equilibrada entre sus tres opciones lo cual significa que un 77 por ciento no cuentan con un plan de comunicación redactado. La respuesta vinculada al plan de marketing varía ligeramente. Un 45 por ciento de los profesionales cuenta con un plan explícito. La normativa general de comunicación, señala un porcentaje similar a los anteriores (32 por ciento "explícita", 78 por ciento "no explícita"). La existencia de submarcas está divida en el seno de los clubes. Un 48 por ciento de los profesionales señala la inexistencia de ellas. El resto se reparte aquellas que están reglamentadas y otras que no. No hay una tendencia clara en este sentido. 


\section{Sí, Sí, No}

de forma explícita aunque no está redactada

\begin{tabular}{lllr}
\hline Misión y Visión & $23 \%$ & $61 \%$ & $16 \%$ \\
\hline Valores & $35 \%$ & $61 \%$ & $4 \%$ \\
\hline Plan Estratégico General & $30 \%$ & $48 \%$ & $22 \%$ \\
\hline Estrategia de COM y MK vinculada & $29 \%$ & $53 \%$ & $18 \%$ \\
\hline Objetivos de cada departamento & $44 \%$ & $44 \%$ & $12 \%$
\end{tabular}

\section{Figura 5. ¿Cuáles son los públicos de mayor importancia? Puede marcar varias respuestas. Fuente: elaboración propia.}

El establecimiento de un mapa de públicos señala también el estado del sector. Un 19 por ciento dispone de un mapa concreto. El resto no tiene dibujado ese mapa de situación. Entre la valoración de esos públicos hay una coherencia y similitud entre los distintos grupos. El gráfico global destaca los abonados (70 respuestas sobre 77), los medios de comunicación (54) y los patrocinadores o clientes (50). A la zaga van los jugadores y los aficionados potenciales. Otros públicos como ex abonados (8), ex clientes (11), agrupaciones de abonados (17) y accionistas (6) y líderes de opinión (16) son los menos importantes para los clubes.

\subsection{La visión crítica de los expertos}

La etapa de contraste de la investigación arroja algunas consideraciones críticas acerca del uso de los procesos de planificación y de la estrategia de identidad corporativa.

Pascual Martínez indica que "no hay una estrategia desde la cual emane todo. Entonces las cosas no se hacen de forma planificada". E interpreta: "El profesional del marketing está muy lejos de la estrategia corporativa. Sí está relacionado con la estrategia de marketing o de branding pero no con la de la corporación, falta un sentido [...] El plan de marketing es el elemento más compartido 45 por ciento".

Cebrián coindice con esta percepción: "Se ve claramente la ausencia de criterios corporativos que deben definirse desde la estrategia dónde en función de los valores, de los ejes de posicionamiento de una marca deportiva, en este caso, tú construyes una estrategia". Carlos Campos reacciona con una opinión similar: "No hay un consciencia de la importancia de la planificación".

Abejón, por su parte, critica esa ausencia de itinerario:

Esta es una de las cosas de las que se adolece el sector de una forma muy importante, no hay planificación. El argumento del club es que no existe planificación porque en dos meses podemos tirar al entrenador; tenemos que cambiarlo y vuelta a empezar. Esa filosofía deportiva, que en principio es errónea, salta al resto de departamentos. Es casi imposible encontrar un club en España que tenga esa planificación. En otros países lo harán mejor o peor, 
pero sí que hay cierto sentido, identidad, filosofía y planificación. Tienen cierta definición de un plan aunque luego se ejecute mejor o peor. Quizá fracasa, pero si no lo tiene seguro que lo hace. La tradición de este país es de tenemos un entrenador y una plantilla y con eso es suficiente.

Lacasa lanza de forma contundente una visión de futuro de acuerdo con este reto:

Ese es el principal problema de las entidades deportivas. Hay que pensar en estratégico y eso supone saber dónde estás y saber dónde quieres ir. Pero eso tan sencillo hay que prepararlo y desarrollarlo de forma sistemática. Uno de los grandes retos del deporte es la planificación estratégica.

Y Alloza, clarividente, considera:

El discurso de los propios clubes es cortoplacista. Ingresar para pagar deudas sin pensar en un mañana. Y más cuando el argumento que deberían utilizar los clubes es un argumento estratégico: 'Yo tengo el potencial en el largo y en el medio plazo para construir una marca potentísima que va a permitir después un negocio recurrente con las empresas porque estoy cubriendo una necesidad que no pueden cubrir con otros actores'. Es que no hay nada equivalente, quizá la música, por lo tanto van a estar obligados a sofisticarse porque se lo exigen las marcas. Evolucionado van a conseguir mejores alianzas, mejor pagadas y además el propio tejido empresarial español va a avanzar por el camino de la creación de marcas. [...\} Como club, tengo el potencial en el largo y en el medio plazo, desde la identidad, para construir una marca potentísima que va a permitir después un negocio.

\section{Conclusiones finales}

Las conclusiones que a continuación se presentan giran en torno a dos elementos enunciados en la hipótesis que guía este trabajo y que una vez desarrollada esta investigación se peude confirmar: la adolescencia de procesos de planificación y la indefinición de una estrategia de identidad corporativa; y la posibilidad de construir marcas poderosas a partir de la indudable atracción que desprende la identidad de un club deportivos.

Resulta evidente que a excepción de los objetivos y planes del área de marketing, los clubes no presentan de forma genérica una redacción explícita de sus bases identitarias, corporativas y estratégicas. La cuestión es especialmente sangrante si el análisis se detiene en la identidad y la filosofía corporativa. No hay un conocimiento global por parte de los profesionales que integran la organización de las metas fijadas y las políticas para alcanzarlas. Tampoco se definen y comparten por escrito las herramientas específicas de planificación básicas en el ámbito de la comunicación corporativa y el marketing como un MÁGICO, un Mapa de Públicos o unas Normas Generales de Comunicación. Por tanto, es indiscutible la ausencia de criterios corporativos que definan la estrategia en función de unos valores y de unos ejes de posicionamiento de marca.

Sin embargo, la relación de los aficionados respecto al club es muy intensa. Tal y como afirma Ángel Alloza:

Los aficionados al deporte tienen una vinculación y un nivel de compromiso brutal. Ese nivel de compromiso es lo que se llama en los libros 'identidad'. Los clubes atesoran una identidad poderosísima que arranca con un relato de los orígenes, siempre está vinculada a la creación de 
historias en el deporte con unos personajes o arquetipos en los que resulta muy fácil construir una relato que sea capaz de generar muchísima más adhesión.

El origen o principio de la vinculación emocional entre una entidad deportiva y sus fans viene dado por los atributos identitarios que la definen. La identidad, junto a la propia competición, es el principal factor de atracción un club. El sociólogo Garry Crawford (2004:20) señala que "ser un fan no es simplemente una etiqueta o una categoría, sino una identidad y un comportamiento". Sin embargo la investigación ha demostrado que este tipo de organizaciones no conciben una definición generalizada de los valores identitarios.

Existe unanimidad en la potencialidad económica que presentan los clubes deportivos como consecuencia de su atracción. Tanto los profesionales participantes en el cuestionario como los expertos entrevistados coinciden en este argumento. Lacasa apunta que "la vinculación del incremento de ingresos con la satisfacción, grado de pertenencia e identificación con los valores del club, por parte del aficionado es absoluta". En cualquier caso, el origen de la oportunidad que las organizaciones deportivas tienen frente a sí recae en la atracción fidelidad o lealtad- que tienen respecto a sus públicos, concretamente sus fans. El vínculo emocional que sostienen club y afición se sustenta en la identidad corporativa, tal y como se ha apuntado a lo largo del trabajo y en la primera estación de la ruta hacia la excelencia.

Alloza señala que este compromiso y relación que debe construir en una marca convencional y que tiene un costo elevado, un club lo incluye en su ADN, en su naturaleza originaria. Considera que si la alta gestión del club evolucionara conseguiría mejores alianzas y mejor remuneradas. La capacidad de generar mayores ingresos es un argumento definitivo a la hora de convencer a los líderes de las organizaciones de la oportunidad de la adopción conceptual e implementación de la filosofía del corporate, aunque bien es cierto que la rentabilidad en la apuesta por la comunicación estratégica vendrá dado en el largo plazo.

Sería recomendable que los clubes asumieran la facultad de gestionar estratégicamente la identidad corporativa a través de las técnicas y herramientas que el corporate ofrece en el ámbito de los recursos y activos intangibles. De este modo serían capaces alcanzar un triple beneficio: la satisfacción de los públicos más implicados, su fidelidad y mejores argumentos para la generación de ingresos económicos. Siguiendo esta línea, Saucedo vislumbra:

Los clubes deben tener esos valores sólidos y los directivos que entrarán y saldrán, porque es ley de vida, tendrían que ser respetuosos con esos valores. Si los directivos fueran capaces de respetar la identidad y los aficionados tendrían mucho ganado y construirían sobre algo estable. 


\section{Referencias bibliográficas}

AlcoBA, Alberto (2010): ¿Quo vadis, deporte? Madrid, Eresma.

Agudo, Ángel y ToYos, Francisco (2003): Marketing del fútbol. Madrid, Pirámide.

AllozA, Ángel y Oliver, Xavier (2009): Atrapados por el consumo. Madrid, Lid Editorial.

ARCEO, Alfredo (2003). "El fútbol y las relaciones públicas en España", en Revista Latinoamericana de Comunicación CHASQUI, no 84, pp. 26-33.

BARAJAS, Angel (2005): El valor económico del fútbol. Radiografía financiera del fútbol español. Pamplona, Eunsa.

CAMPoS, Carlos (1997): Marketing y patrocinio deportivo. Madrid, Gestión y Promoción.

CAmpos, Carlos (2006): Estrategia de saneamiento del deporte profesional. Sevilla, Wanceulen Editorial Deportiva.

CASTELlS, Manuel (1997): La era de la información, vol I. Madrid, Alianza.

CAPRIOTTI, Paul (2009): Branding corporativo: fundamentos para la gestión estratégica de la identidad corporativa. Santiago de Chile, Libros de Empresa.

CHADWICK, Simon; y Beech, John (2004): The business of the sport: text and cases on strategy and management. Essex, Prentice Hall.

CHADWICK, Simon; y Beech, John (2010): Managing Football an international perspective. Oxford, Butterworth - Heinemann.

CostA, Joan (2003): Imagen corporativa en el siglo XXI. Buenos Aires, La Crujía.

CostA, Joan (2008): El dircom hoy. Barcelona, Costa Punto Com Editor.

CRAWFORD, Gerry (2004): Consuming sport. Londres, Loutedge.

FERRAND, Alain, y MCARTHY, Scott (2009): Marketing the sports organization. Building networks and relationships. Oxon, Routledge.

GARCÍA, Domingo (2004): Ética empresarial: del diálogo a la confianza. Madrid, Trotta.

GINESTA, Xavier (2009): Las tic y el deporte: un análisis de la primera división española de fútbol. Bellaterra (Tesis Doctoral, Universitat de Barcelona. Departamento de Periodismo i Ciencias de la Comunicación).

GIULIANOTTI, Robert (2005): "Sports Spectators and the Social Consequences of Commodifications: Critical Perspectives from Scottish Football", en Journal of Sport and Social Issues, vol. 29, no 3 (abr., 2005), pp. 386-410.

GómEZ, Saandalio, KASE, Kimio y URRUTIA, Ignacio (2010) Value creation and sport management. London, Cambridge. 
HOPWOOD, Maria (2005): "Applying the public relations function to the business sport", en International Journal of Sports Marketing and Sponsorship. vol. 6, no 3 (abr., 2005), pp. 164-181.

HOPWOOD, Maria (2010): "Sport marketing public Relations" en Hopwood, M., Kitchin, $\mathrm{P}$ y Skinner,J. Sport public relations and communication. Oxford, Butterwoth-Heinemann.

LóPEZ, Rafael (2000): La comunicación: la clave del bienestar social. Madrid, Ediciones El Drac.

LóPEZ, Rafael (2001): Las agencias de publicidad: evolución, posicionamiento y futuro. Castellón, Publicaciones de la Universitat Jaume I.

LóPEZ, Isabel y Sotillo, Sandra (2009): "La gestión de los recursos intangibles empresariales", en Cuadernos de gestión del conocimiento empresarial, no 11 (abr., 2009), pp. 72-84.

MARÍn, Joaquín (ed.) (2009): Imagen, comunicación y deporte. Una aproximación teórica. Sevilla, Visión Libros.

MASTERMAN, Guy (2009): Strategic Sports Event Management: Olympic Edition. London, Elsevier-Butterworth Heinemann.

Mediavilla, Gerardo (2001): Comunicación corporativa en el deporte. Madrid, Gymnos.

MoragaS SpÀ, Miguel de (2007): "Comunicación y deporte en la era digital" [artículo en línea]. Barcelona: centre d'estudis olímpics uab. http://olympicstudies.uab.es/pdf/wp107_spa.pdf (consultado el: 11/02/11)

MORALES, Francisca (2007). La comunicación planificada (Tesis Doctoral, Universitat de Barcelona. Departament de Periodismo i Ciencias de la Comunicació).

MuLLIN, James; HARDY, Stephen Y HUTTON, William (1992): Marketing deportivo. Barcelona, Paidotribo.

Mut, Magda. (2010). "Rumbo tras la perspectiva comparada del director de comunicación", en Revista Académica del Foro Iberoamericano sobre Estrategias de Comunicación, vol. 4, no 14. (oct., 2010), pp. 25 -39.

OlABE, Fernando. (2009): "La comunicación no convencional en los clubes de fútbol", en Pensar la Publicidad, vol 3, no 1 (oct., 2009), pp.121-138.

PARKS, Janet B. y QUATERMAN, Jerome (1998): Contemporary sport management. Champaign, Human Kinetics.

Pedersen, Paul; Miloch, Kimberly y Laucella, Pamela (2007): Strategic sport comunication. Champaign, Human Kinetics.

PÉREZ, Alberto (2001): Estrategias de comunicación. Barcelona, Ariel.

RODRÍGUEZ, Inma. (Coord) (2007). Estrategias y técnicas de comunicación. Una visión integrada del marketing. Barcelona, UOC. 
Slotd, G.Clayton, DitTmore, Stephen.W. Branvold, Scott.E. (2006): Sport public relations. Managing organitational communication. Champaign, Human Kinetics.

VERDÚ, Vicente (2009): El capitalismo funeral. Barcelona, Anagrama .

[Recibido: 23 de enero de 2013. Aceptado: 26 de septiembre de 2013]. 